\title{
Grain Boundary Wetting in Zn Bicrystals by a Sn-based Melt
}

\author{
Vladimir Murashov ${ }^{1, a}$, Boris Straumal ${ }^{2, b}$ and Pavel Protsenko ${ }^{1, c}$ \\ ${ }^{1}$ Department of Chemistry, MSU, 119992 Moscow, Russia \\ ${ }^{2}$ Institute of Solid State Physics, Russian Academy of Sciences, 142432 Chernogolovka, Russia \\ amva84@mail.ru, bstraumal@issp.ac.ru, ${ }^{\mathrm{a}}$ protsenko@colloid.chem.msu.ru
}

Keywords: Interfacial energy, grain boundary energy, wetting, grooving.

\begin{abstract}
The kinetics and morphology of the grain boundary grooving of $\mathrm{Zn}$ bicrystals with $16^{\circ}$ $<1010>$ tilt $\mathrm{GB}$ by $\mathrm{Sn}(\mathrm{Zn})$ melt has been studied at $325^{\circ} \mathrm{C}$ in equilibrium conditions in vacuum. It is shown that grooving process is interface controlled at least on the first stage. Groove walls mobility is evaluated. Changing of GB grove shape from "faceted walls" corner for annealing time $<78 \mathrm{~h}$ to concave "Mullins type" groove for annealing time $>78 \mathrm{~h}$ at the same experimental temperature was observed for the first time.
\end{abstract}

\section{Introduction}

The properties of advanced polycrystalline materials depend critically on the properties of interfaces such as grain boundaries (GBs). In particular, interaction between GBs and melts in structural materials may be the reason of deterioration of mechanical properties and failure. Interaction of saturated liquid metal (LM) melt with a GB in a solid metal is thoroughly studied due to both application (compatibility of liquid metal coolants, solders and coatings with structural materials) and fundamental (mechanisms of LM penetration into GB and transport of system components) reasons. Dihedral angle $\varphi$ formed in the vicinity of the GB groove is known to be tightly linked

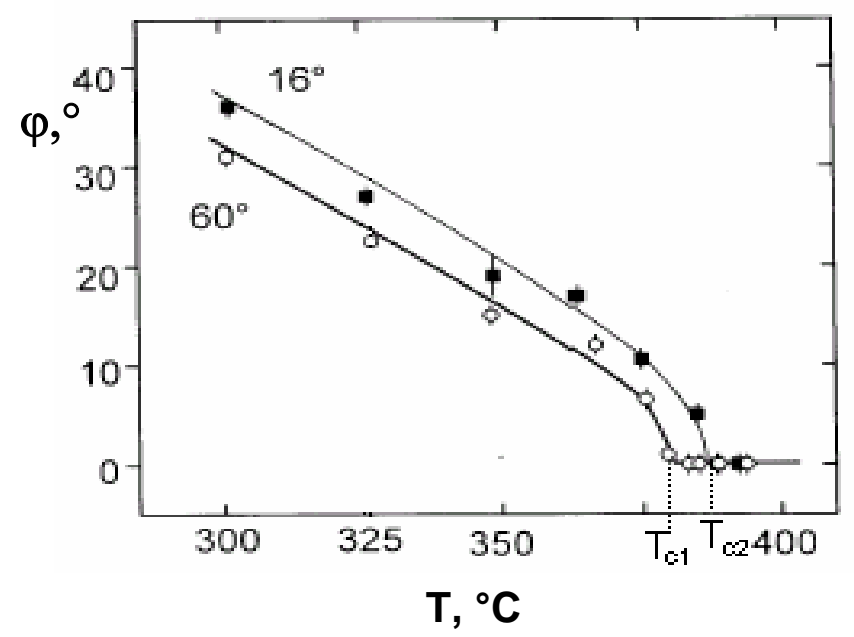

Fig. 1 Dependence on dihedral angle $\varphi$ on temperature for $\mathrm{Zn}$ bicrystal with $16^{\circ}$ and $60^{\circ}$ $<1010>$ tilt GB in contact with Zn-Sn melt [1] with the mechanism of GB grooving: at high $\varphi>60^{\circ}$ the GB grooving is limited by volume diffusion through the liquid and at $\varphi=0^{\circ}$ complete wetting of GB by liquid phase occurs (GB wetting rate is limited by the process at the groove tip, at least on early stage). Present study is focused on the case $0<\varphi<60^{\circ}$ which is much less studied. Selection of liquid $\mathrm{Sn}(\mathrm{Zn}) /$ solid $\mathrm{Zn}$ system was caused by its potential to replace traditional $\mathrm{Pb}-\mathrm{Sn}$ solders and well established $\varphi(T)$ dependence determined in [1]. Bicrystals were selected instead of polycrystals in order to avoid ambiguity linked to different energetics of various GBs in polycrystals.

\section{Experimental}

$\mathrm{Zn}$ bicrystals with $16^{\circ}<1010>$ tilt GB were grown from $\mathrm{Zn}$ of 99,999 wt.\% purity by a modified Bridgman technique [2]. After growing bicrystals were cut by spark erosion into $10 \times 12 \mathrm{~mm}$ pieces. For the wetting experiments the $\mathrm{Zn}$ bicrystals were etched for $10-30 \mathrm{~s}$ in $\mathrm{HNO}_{3}$ in order to remove the deformed layer and brought in contact with a small amount of melted Sn (analytical grade 
purity) in the region of GB intersection with sample surface. Annealing temperature has been chosen to be $325{ }^{\circ} \mathrm{C}$ which corresponds to $\varphi \approx 30^{\circ}$ according to [1]. Samples were annealed in evacuated ampoules for 0.5 to $242 \mathrm{~h}$ (residual pressure $0.1 \div 1 \mathrm{~Pa}$ ) and water quenched. After quenching samples were cut perpendicular to GB and former solid-liquid planes, ground and polished to $1 \mu \mathrm{m}$ diamond paste. Geometrical parameters of GB grooves were measured using light microscope Neophot 21.
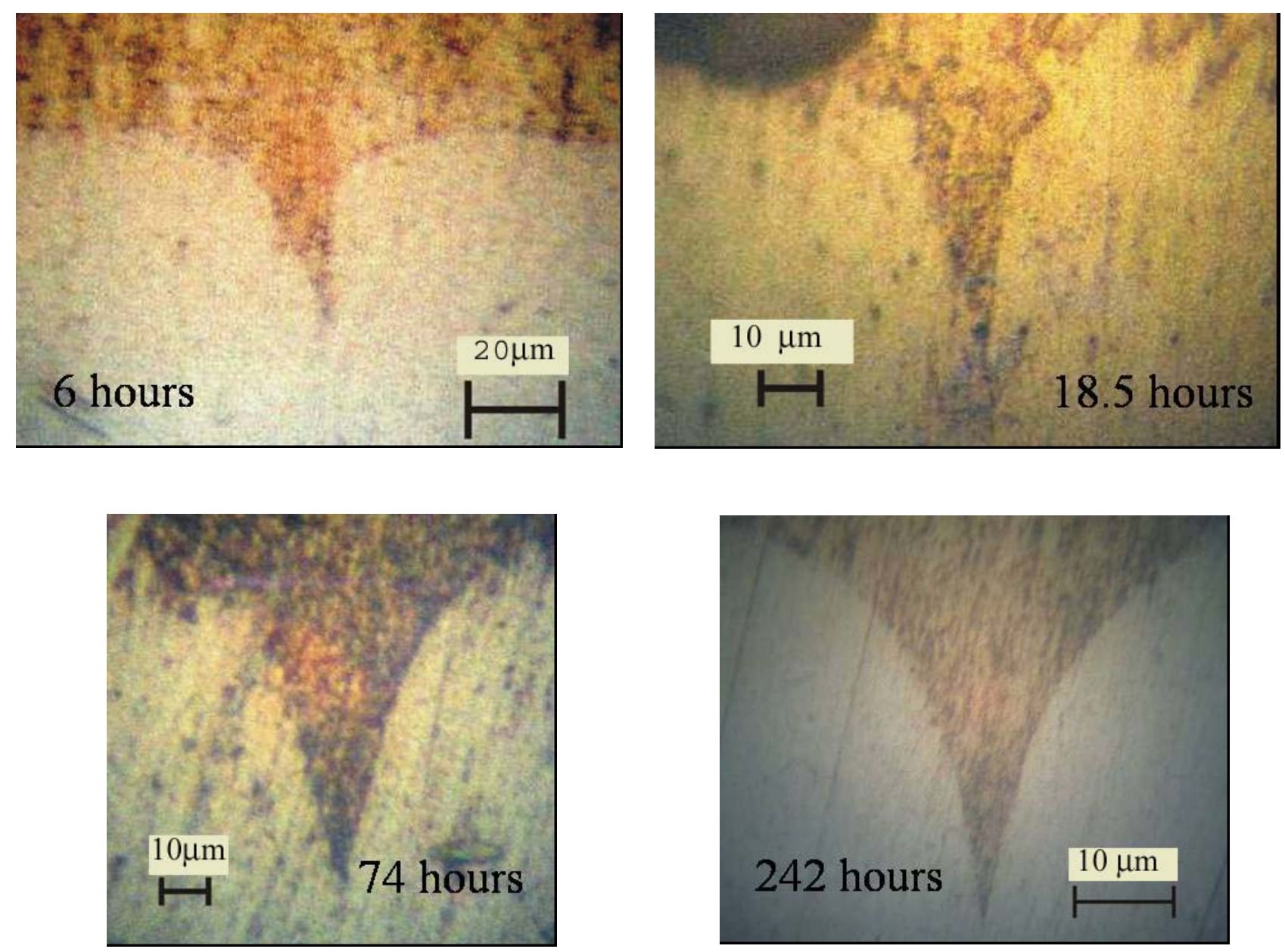

Fig. $2 \mathrm{Zn} \mathrm{GB}$ grooved by $\mathrm{Sn}(\mathrm{Zn})$ melt at $325^{\circ} \mathrm{C}$ for $6,18.5,74$ and $242 \mathrm{~h}$. Section perpendicular to SL interface, light microscopy.

\section{Results and discussion}

Optical photographs of obtained GB grooves are presented in Fig. 2. In accordance with previous data [1] $\mathrm{Sn}(\mathrm{Zn})$ melt does not wet $16^{\circ}<1010>$ tilt $\mathrm{GB}$ at $325^{\circ} \mathrm{C}$. In Fig. 3a dihedral angle as a function of time is presented. It was found to be equal to $26 \pm 1^{\circ}$ and did not change during annealing process. This fact confirms assumption that interface and grain boundary energies are constant during experiment.

On the contrary, significant change in grooves morphology with time was observed. For annealing time less than $78 \mathrm{~h}$ groove walls were flat. In the samples subjected to longer annealing flat regions disappear and both sides of the groove were convex toward the melt. It can be seen from fig. 3b. that groove growing kinetics obeys the $d \sim t^{1 / 2}$ law. In order to determine the factors affecting growing kinetics, the experimental penetration rate was compared with one predicted by Mullins for volume diffusion controlled GB grooving [3]. 


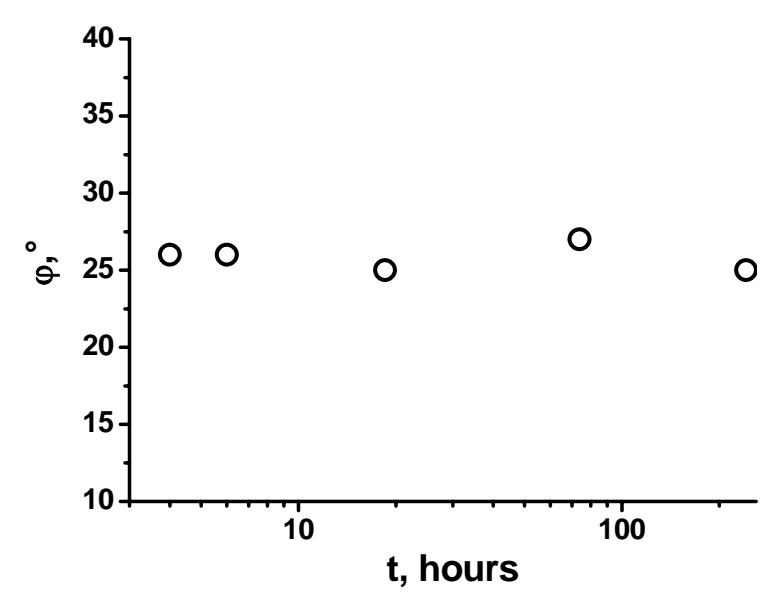

a

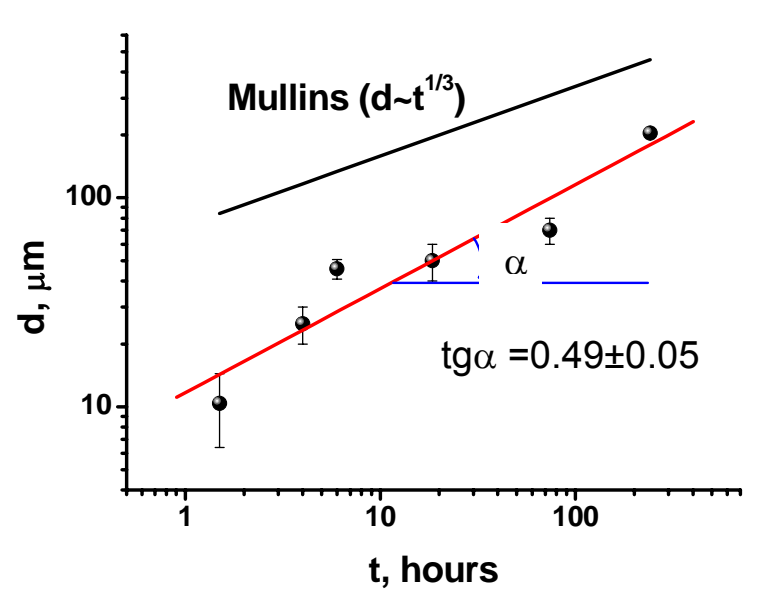

b

Fig.3 Dependence of contact angle (a) and penetration depth (b) on annealing time.

$$
d=\frac{1.01}{\operatorname{tg} \varphi / 2}\left(\frac{C_{0} \sigma_{s l} V_{m}^{2} D}{R T} t\right)^{1 / 3}
$$

Following values were used for quantitative calculations: equilibrium concentration of $\mathrm{Zn}$ in $\mathrm{Sn}$ melt at $325^{\circ} \mathrm{C} C_{0}=5.13 \cdot 10^{4} \mathrm{~mol} / \mathrm{m}^{3}[4], \sigma_{\mathrm{sl}}=150 \mathrm{~J} / \mathrm{m}^{2}$ at $200^{\circ} \mathrm{C}$ and $\mathrm{d} \sigma_{\mathrm{sl}} / \mathrm{d} T=-0.29 \mathrm{~J} / \mathrm{m}^{2}[5], V_{\mathrm{m}}=$ $9.2 \cdot 10^{-6} \mathrm{~m}^{3} / \mathrm{mol}, D_{\mathrm{Zn}(\mathrm{Sn})}=1 \cdot 10^{-8} \mathrm{~m}^{2} / \mathrm{sec}$ [6]. Calculated penetration depth is plotted in Fig. $3 \mathrm{~b}$ by solid line and it is significantly higher than experimental one.

On the basis of obtained morphological and kinetic data we suppose that limiting stage of groove growth is the dissolution of faceted grove walls. The growth of faceted groove controlled by dissolution was considered in details by Rabkin [7]. According to proposed model the migration rate of the facets is proportional to the driving force, with a coefficient of proportionality, $M$, defined as the facet mobility.

$$
M=\frac{A \sin \frac{\varphi}{2}}{2 \sigma_{s l}\left(1-\sin \frac{\varphi}{2}\right)} .
$$

In order to calculate the facet mobility in our case the following values were used: dihedral angle $\varphi$ $=26^{\circ}, \sigma_{\mathrm{sl}}=150 \mathrm{~J} / \mathrm{m}^{2}$ at $200{ }^{\circ} \mathrm{C}$ and $\mathrm{d} \sigma_{\mathrm{sl}} / \mathrm{d} T=-0,29 \mathrm{~J} / \mathrm{m}^{2}$ [5]. $A$ is a constant of groove growing rate:

$$
d^{2}=A t \text {. }
$$

Calculated facet mobility was equal to $6 \cdot 10^{-14} \mathrm{~m} /(\mathrm{Pa} \cdot \mathrm{s})$.

It should be noted that changes of groves shape at longer annealing time to "Mullins type" did not lead to kinetics change from $d \sim t^{1 / 2}$ to $d \sim t^{1 / 3}$. Nevertheless such a change could be an indication of gradually changing mechanism from interface dissolution control to volume diffusion control as diffusion paths becomes longer with grove deepening. Further experiment with longer annealing times are in progress in order to verify this hypothesis.

The other important expectation based on our experimental results is that in some cases GB grove shape (macroscopic flat facet's or concave smooth walls) dictated not by degree of solidliquid interface anisotropy (as it is commonly accepted) but by limiting stage of grooving process: when diffusion paths are short dissolution rate controls grooving and walls are flat and for deeper grooves diffusion becomes a limiting stage and walls are convex. 


\section{Conclusions}

Described above analysis of GB grooving in $\mathrm{Zn}$ bicrystal / $\mathrm{Sn}(\mathrm{Zn})$ melt system could be summarized as follows:

1. The dihedral angle does not depend on the annealing time which is an indication of constansy of GB energetics and chemistry

2. When the time of annealing is less than 78 hours:

- $\quad$ GB grooves are faceted;

- $\quad$ kinetics of grain boundary grooving obeys $\mathrm{d} \sim \mathrm{t}^{1 / 2}$ law;

- depth of the groves is less than predicted by Mullins model, that denotes kinetic limitations. The facet mobility is $6 * 10^{-14} \mathrm{~m} /\left(\mathrm{Pa}^{*} \mathrm{~s}\right)$.

3. The grow of convex groove follows the grow of faceted groove during the annealing. The change of grooves morphology of with time of annealing more 78 hours that denotes that for the very long annealing time the diffusion will probably become the limiting kinetic factor.

\section{Acknowledgements}

The investigations were partly supported by the German Federal Ministry for Education and Research (contract RUS 04/014), INTAS (contract 03-51-3779) and Russian Foundation for Basic Research (contract 05-02-16528).

\section{References}

[1] B.B. Straumal, W. Gust and T. Watanabe: Mater. Sci. Forum. Vols. 294-296 (1999), p. 411

[2] B.B. Straumal: Grain boundary phase transitions (Nauka publishers, Moscow 2003)

[3] W. Mullins Trans. Metal. Soc. AIME Vol. 218 (1960), p. 354

[4] N. Eustathopoulos: Int. Metals Rev. Vol. 28 (1983), p. 189

[5] T.B. Massalski et al. (eds): Binary alloy phase diagrams (ASM International, Materials Park 1993)

[6] F.H. Huang and H.B. Hungtington: Phys. Rev. B Vol. 9 (1974), p. 1479

[7] D. Chatain, E. Rabkin, J. Derenne and J. Bernardini: Acta mater. Vol. 49 (2001), p. 1123 